\title{
Cross Reactivity
}

National Cancer Institute

\section{Source}

National Cancer Institute. Cross Reactivity. NCI Thesaurus. Code C63261.

Problem associated with the deg ree to which an antibody or antigen participates in cross reactions. 